\title{
Lenti in red: progress in gene therapy for human hemoglobinopathies
}

\author{
Christof von Kalle, ${ }^{1}$ Christopher Baum, ${ }^{1,2}$ and David A. Williams ${ }^{1}$
}

${ }^{1}$ Division of Experimental Hematology, Cincinnati Children's Hospital Medical Center and University of Cincinnati College of Medicine, Cincinnati, Ohio, USA. 2Department of Hematology/Oncology, Hanover Medical School, Hanover, Germany.

\begin{abstract}
Hemoglobinopathies are caused by abnormal structure or synthesis of hemoglobin chains and represent serious monogenic disorders. A new study demonstrates that lentiviral vectors can express clinically relevant levels of human transgenic $\beta$-globin in red cells of xenografted mice (see the related article beginning on page 953 ). While some safety concerns must be addressed, this study is an important step toward potential clinical trials of gene therapy for hemoglobinopathies.
\end{abstract}

\section{Gene transfer technology evolves, but with side effects}

For many researchers, the use of gene transfer technology, in which genetic material (DNA) is transferred into the patient's cells to compensate for a gene deficiency, has been a major goal. In the early 1980s, the development of murine leukemia virus-based (MLV-based) retrovirus vectors, derived from naturally evolved viruses capable of transferring their genetic materials with high efficiency into the host cells, facilitated the genetic manipulation of murine hematopoietic stem cells $(1,2)$. The application of gene transfer technology to treat human disease has since been much anticipated (3).

Early investigators contemplated the use of gene transfer for the treatment of human monogenic diseases in which the regulation of the defective gene was relatively straightforward, for example, not subject to lineage- or developmental-specific regulation. Accordingly, SCID, due to deficiency of adenosine deaminase, was the first human disease for which a gene therapy clinical trial was initiated $(4,5)$. The most common form of the disorder is X-linked SCID (X-SCID), which is caused by mutations in or deficiency of the common $\gamma$ chain of the IL-2 receptor, an essential component of 5 cytokine receptors necessary for the development of T and NK cells. SCID can be fatal during the first year of life because of

Nonstandard abbreviations used: MLV, murine leukemia virus; X-SCID, X-linked SCID.

Conflict of interest: The authors have declared that no conflict of interest exists.

Citation for this article: J. Clin. Invest. 114:889-891 (2004). doi:10.1172/JCI200423132. severe, recurrent infections. However, a gene transfer approach, in which autologous hematopoietic stem cells transduced in vitro with the $\gamma$ chain using a retroviral vector, has been shown to restore the immune system of SCID patients (6). These studies were halted in 2003 after the development of insertional leukemogenesis in two patients in this trial (7). The discovery that the vector-based enhancer and promoter dysregulated the expression of $L M O 2$, a known protooncogene, in these leukemias was followed by the observation that, in a broader context, MLV-based vectors show a preference for insertions in promoter-proximal regions of active genes (8). These events led to an urgent search for safer vectors for use in human gene therapy and pointed to the need to develop vector systems that more precisely mimic physiologic expression patterns. Because of their ability to be transduced after a very short period of ex vivo culture, the absence of a strong promoter and enhancer in the vector long-terminal repeat element, and reduced propensity to integrate in promoter-proximal gene regions (8), lentivirus vectors are considered by some as potentially safer than retroviral vectors, and this has led to further acceleration of this vector system.

\section{$\beta$-globin gene transfer}

Of great importance to the successful clinical transfer of globin genes is a thorough understanding of the cis-regulatory elements that affect globin gene expression, and this understanding has evolved from the work of many laboratories (9-11). In the case of human $\beta$-globin, these efforts resulted in initial successes in obtaining curative levels of lineage-specific $\beta$-globin expression in the progeny of murine stem cells by two research groups $(12,13)$. In this issue of the JCI, Imren et al. (14) demonstrate that human stem and progenitor cells transduced with a lentiviral vector encoding a $\beta$-globin transgene and engrafted into an immunodeficient mouse model express human $\beta$-globin in their erythroid progeny at levels that would be anticipated to improve the phenotype of sickle cell anemia or $\beta$-thalassemia in affected individuals. However, Imren et al. present evidence for frequent proviral integration of the $\beta$-globin transgene within genes that play crucial regulatory roles in hematopoiesis, reinforcing a need for additional studies to ensure the long-term safety of this approach in gene therapy protocols.

\section{Vector regulation and expression}

The lentiviral transgene used by Imren et al. (14) represents one of the most advanced and powerful $\beta$-globin expression vectors available to date. However, even with this vector, two copies were needed per cell for high-level transgene expression. The authors speculate that further optimizations will be required for clinical use in order to achieve even higher protein expression from a single gene copy. This is an important goal, given that the integration pattern near genes important for regulation of hematopoiesis observed in this study indicates a possibility of potential long-term toxicities caused by the dysregulation or disruption of such genes. In addition, the vector may have to be modified to include a selectable marker gene (such as a gene mediating cytotoxic drug resistance in hematopoietic stem cells) because in its present form, the efficiency of gene transfer may not be high enough to modify a sufficient number of stem cells (15). Otherwise, nonselectable globin vectors might only be useful if the intended recipient undergoes strong myeloablative conditioning prior to transplantation of gene-modified cells. Coexpression of transcriptionally targeted $\beta$-globin and a 


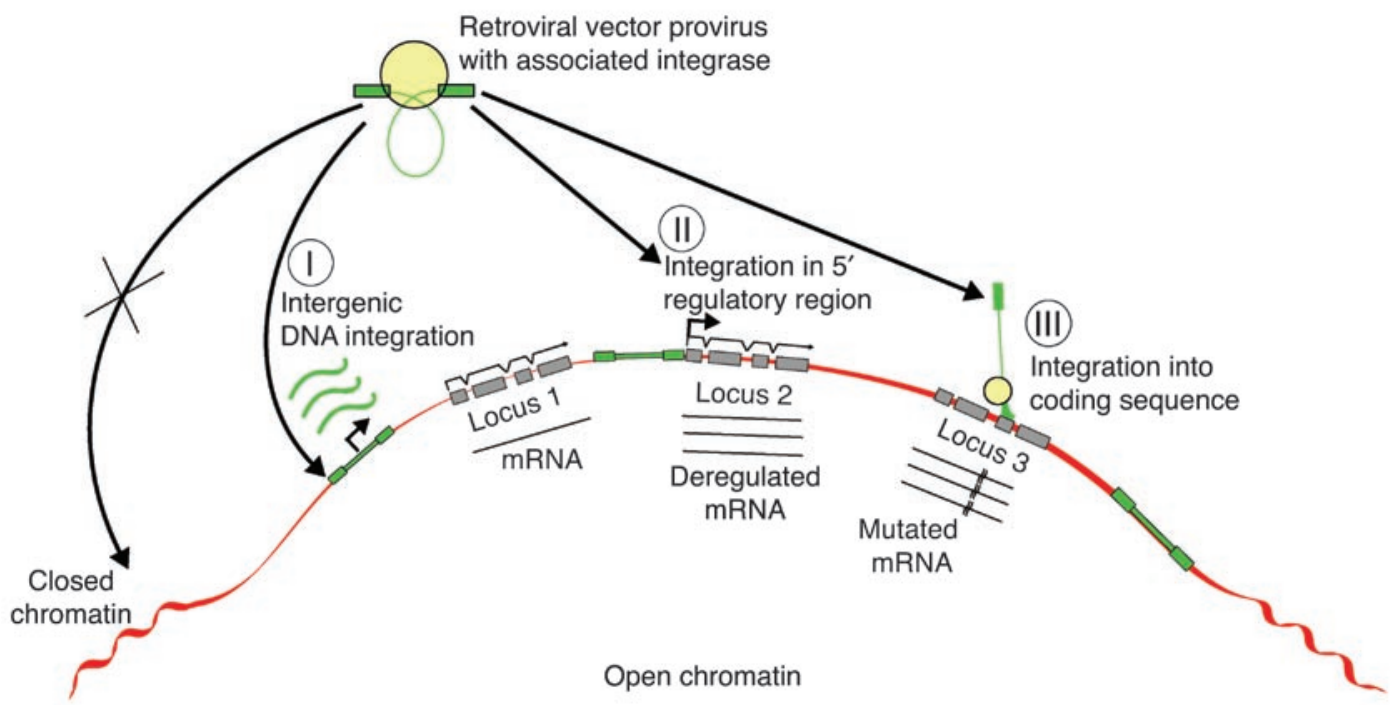

Figure 1

Distribution of retrovirus integration into the cellular genome. Lenti- and other retrovirus vector integration frequently occurs in the vicinity of expressed genes. Integration in intergenic locations, especially at distances of more than 100 kbp's away from any known gene (I), are least likely to affect the transcription of known genes. MLV-derived vectors appear to have a preference for integrating close to the start of transcription (II), a location especially likely to alter the expression of adjacent endogenous genes. Lentivirus vectors appear to more frequently integrate within the gene (III), a location less likely to affect genetic regulation but potentially more likely to cause disruptions of the mRNA transcript of the endogenous gene.

selectable marker gene acting at the level of the hematopoietic stem cell is possible (16); however, the expression properties and infectious titers of such dual-function vectors are not yet optimal, and few studies have focused on the potential side effects of in vivo selection and consequent forced expansion on the stem cell compartment.

Can we envisage further vector improvements? There are many options for finetuning of vector performance. These range from enhancer and promoter design (e.g., incorporation of extended enhancer elements (17) or use of bidirectional promoters to drive two globin sequences) to the development of modules affecting post transcriptional regulation of protein expression, and protein engineering. As these are all complementary mechanisms, even a small gain of function achieved as a result of modifications to each would be meaningful. Alternatively, attempts to further increase the safety of the vector's integration pattern, thus reducing the impact of multiple insertions in single cells, would be most useful. This area of research is highly active, and we envisage a profoundly increased repertoire of gene delivery technologies in the not-too-distant future (18, 19). Irrespective of these considerations, the results presented by Imren et al. (14) are promising and may be even better in $\beta$-thalassemic cells, in which the competi- tion with the expression of endogenous $\beta$-globin is reduced (20). Moreover, as the study of erthyropoiesis is currently carried out primarily in vitro or in immunodeficient mouse models - neither of which represents ideal models of the human disease - there is some hope that the existing vectors will work even better in human subjects.

\section{Vector integration in relevant hematopoietic genes}

In addition to providing proof of vector function, Imren et al. (14) have sought to study the distribution of vector insertions present in the engrafting cells of their model for the dual purposes of visualization of the number of repopulating clones present and the distribution of genomic vector insertion in an in vivo model. Although their experimental approach was limited by the number of insertion events that could be analyzed, the insertion data presented suggest two interesting interpretations. First, because genetic correction has been achieved in multiple engraftable clones, in a clinical application, a multiclonal or polyclonal correction of stem cells could potentially be achieved with this vector. With our current understanding, this would be favorable for the long-term stability of any genetic correction. Second, although the observations are necessarily of somewhat limited size for a rigorous statistical study of insertion site distribution, Imren et al. found preferential integration of lentiviral vectors into introns of actively expressed genes. These data suggest that the distribution of lentivirus vector integrations into hematopoietic repopulating cells may be similar to that shown in earlier studies in cell lines $(10,21)$. After the description of the side effects of insertional gene activation observed in the highly effective X-SCID gene therapy trial (7), investigators' attention has focused on integration site characteristics for all insertional vector systems. In the past, the risk of insertional mutagenesis had been considered small to negligible for replication-incompetent vectors derived even from mutagenic retroviruses. Insertions of vectors were thought to be mostly randomly distributed throughout the genome as single copies, and the number of cells with insertions was considered to be small, making the risk of untoward mutagenic events highly unlikely. More recent studies have demonstrated that the insertion of both MLV retrovirus and lentivirus vectors is nonrandomly distributed in association with actively expressed genes (Figure 1) (8, 21 ). On the basis of an apparent overrepresentation of insertions in genes that are associated with the regulation of proliferation and differentiation, Imren et al. reason 
that the integration distribution noted in their study may be cause for concern, as it may suggest a risk for gene mutation and subsequent disruption of gene function after the application of such vectors.

Care has to be taken not to overinterpret this observation. While it seems prudent to not entirely exclude the possibility that HIV-derived vectors may have some mutagenic potential, HIV infection itself has not been associated as a mutagen with the development of cancer. Lentivirus vectors may lack the 2 mechanisms by which their wild-type parent virus may effectively selflimit any potentially transforming mutagenesis: immunogenicity and the ability to limit the infected cell's life span. However, lentivirus globin vectors also lack the highly effective, long-terminal repeat promoter and enhancer combination that appears to be the main cause of MLV and MLV-based vector-associated insertional oncogene activation. Moreover, neither $\alpha$ - nor $\beta$-globin can be envisaged to cause oncogenic signal alterations if ectopically expressed.

Thus any consideration that insertions of a globin lentivirus or other type of integrating vector with red cell-specific expression could be a transforming mutagen is entirely theoretical at this point. Such side effects might be very rare, as they have not been reported in mouse models of globin gene therapy. However, although protooncogene mutations involving activity of the internal vector promoter and enhancer might be restricted to erythroblasts, these cells are not entirely resistant to transformation, especially when tumor suppressor genes are disrupted (22). On the other hand, monoallelic gene disruption by globin vectors could also affect more primitive progenitors. While potential phenotypic consequences, especially of multiple-hit insertions, need to be investigated in a model system adjusted for that purpose, in the absence of any real proof of untoward side effects, such considerations should not preclude the development of promising vector applications such as the one described here by Imren et al. (14) for the treatment of debilitating or life-threatening disorders.

In summation, gene therapy may offer an effective therapy for hemoglobinopathies if key issues associated with this approach can be solved, including potent and persistent expression of the corrective transgene in red cells after transduction into hematopoietic stem cells, sufficient contribution of corrected cells to long-term hematopoiesis, and absence of mutagenic complications that might result from the imprecision of gene insertions using current vector systems.

\section{Acknowledgments}

We thank Shawn Wheeler and Eva Meunier for manuscript assistance.

Address correspondence to: David A. Williams, Cincinnati Children's Hospital Medical Center, 3333 Burnet Avenue, Mail Location 7013, Cincinnati, Ohio 45229, USA. Phone: (513) 636-0364; Fax: (513) 636-3768; E-mail: David.Williams@cchmc.org.

1. Williams, D.A., Lemischka, I.R., Nathan, D.G., and Mulligan, R.C. 1984. Introduction of new genetic material into pluripotent haematopoietic stem cells of the mouse. Nature. 310:476-480.

2. Joyner, A., Keller, G., Phillips, R.A., and Bernstein, A. 1983. Retrovirus transfer of a bacterial gene into mouse haematopoietic progenitor cells. Nature. 305:556-558

3. Orkin, S.H. 1986. Molecular genetics and potential gene therapy. Clin. Immunol. Immunopathol. 40:151-156

4. Blaese, R.M., et al. 1995. T lymphocyte-directed gene therapy for $\mathrm{ADA}^{-}$SCID: initial trial results after 4 years. Science. 270:475-480.

5. Kohn, D.B., et al. 1998. T lymphocytes with a normal ADA gene accumulate after transplantation of transduced autologous umbilical cord blood CD34+ cells in ADA-deficient SCID neonates. Nat. Med. 4:775-780.

6. Hacein-Bey-Abina, S., et al. 2002. Sustained correction of $\mathrm{X}$-linked severe combined immuno- deficiency by ex vivo gene therapy. N. Engl. J. Med. 346:1185-1193.

7. Hacein-Bey-Abina, S., et al. 2003. LMO2-associated clonal $\mathrm{T}$ cell proliferation in two patients after gene therapy for SCID-X1. Science. 302:415-419.

8. Wu, X., Li, Y., Crise, B., and Burgess, S.M. 2003. Transcription start regions in the human genome are favored targets for MLV integration. Science. 300:1749-1751.

9. Persons, D.A. 2003. Update on gene therapy for hemoglobin disorders. Curr. Opin. Mol. Ther. 5:508-516.

10. Sadelain, M. 2004. Globin gene transfer as a potential treatment for the beta-thalassaemias and sickle cell disease. Vox Sang. 87(Suppl. 2):235-242.

11. Li, Q., Peterson, K.R., Fang, X., and Stamatoyannopoulos, G. 2002. Locus control regions. Blood. 100:3077-3086.

12. May, C., et al. 2000. Therapeutic haemoglobin synthesis in beta-thalassaemic mice expressing lentivirus-encoded human beta-globin. Nature. 406:82-86.

13. Pawliuk, R., et al. 2001. Correction of sickle cell disease in transgenic mouse models by gene therapy. Science. 294:2368-2371.

14. Imren, S., et al. 2004. High-level $\beta$-globin expression and preferred intragenic integration after lentiviral transduction of human cord blood stem cells. J. Clin. Invest. 114:953-962. doi:10.1172/ JCI200421838.

15. Moritz, T., and Williams, D.A. 2002. Transfer of drug resistance genes to hematopoietic precursors. In Encyclopedia of Cancer. 2nd edition. J.R. Bertino, editor-in-chief. 433-447.

16. Persons, D.A., et al. 2003. Successful treatment of murine beta-thalassemia using in vivo selection of genetically modified, drug-resistant hematopoietic stem cells. Blood. 102:506-513.

17. Hanawa, H., et al. 2004. Extended beta-globin locus control region elements promote consistent therapeutic expression of a gamma-globin lentiviral vector in murine beta-thalassemia. Blood. doi:10.1182/ blood-2004-03-0863.

18. Baum, C., et al. 2003. Side effects of retroviral gene transfer into hematopoietic stem cells. Blood. 101:2099-2114.

19. Bushman, F.D. 2003. Targeting survival: integration site selection by retroviruses and LTR-retrotransposons. Cell. 115:135-138.

20. Puthenveetil, G., et al. 2004. Successful correction of the human beta-thalassemia major phenotype using a lentiviral vector. Blood. doi:10.1182/blood2004-04-1427.

21. Schroder, A.R., et al. 2002. HIV-1 integration in the human genome favors active genes and local hotspots. Cell. 110:521-529.

22. von Lindern, M., et al. 2001. Leukemic transformation of normal murine erythroid progenitors: $\mathrm{v}$ - and c-ErbB act through signaling pathways activated by the EpoR and c-kit in stress erythropoiesis. Oncogene. 20:3651-3664. 